\title{
Post-Lie Algebras and Isospectral Flows
}

Kurusch EBRAHIMI-FARD ${ }^{\dagger^{1}}$, Alexander LUNDERVOLD ${ }^{\dagger^{2}}$, Igor MENCATTINI $\dagger^{3}$ and Hans Z. MUNTHE-KAAS ${ }^{\dagger^{4}}$

$\dagger^{1}$ ICMAT, C/ Nicolás Cabrera 13-15, 28049 Madrid, Spain

E-mail: kurusch@icmat.es

URL: http://www.icmat.es/kurusch/personal

$\dagger^{2}$ Department of Computing, Mathematics and Physics, Faculty of Engineering, Bergen University College, Postbox 7030, N-5020 Bergen, Norway

E-mail: alexander.lundervold@gmail.com

URL: http://alexander.lundervold.com

$\dagger^{3}$ Instituto de Ciências Matemáticas e de Computação, Universidade de São Paulo, Campus de São Carlos, Caixa Postal 668, 13560-970 São Carlos, SP, Brazil

E-mail: igorre@icmc.usp.br

$\dagger^{4}$ Department of Mathematics, University of Bergen, Postbox 7803, N-5020 Bergen, Norway

E-mail: hans.munthe-kaas@math.uib.no

URL: http://hans.munthe-kaas.no

Received August 13, 2015, in final form November 16, 2015; Published online November 20, 2015 http://dx.doi.org/10.3842/SIGMA.2015.093

Abstract. In this paper we explore the Lie enveloping algebra of a post-Lie algebra derived from a classical $R$-matrix. An explicit exponential solution of the corresponding Lie bracket flow is presented. It is based on the solution of a post-Lie Magnus-type differential equation.

Key words: isospectral flow equation; $R$-matrix; Magnus expansion; post-Lie algebra

2010 Mathematics Subject Classification: 70H06; 17D99; 37J35

\section{Introduction}

Isospectral flows and the corresponding Lax type equations play an important role in the theory of dynamical systems, both in finite and infinite dimensions. See [1, 12]. They appear together with a large supply of conserved quantities for the original dynamical system. In the finite-dimensional case, i.e., for systems with a finite number of degrees of freedom, the Lax representation may correspond to the Hamiltonian representation of the dynamical system in terms of Euler-type equations on the coadjoint orbits of a suitable Lie group $G$. Writing $\mathfrak{g}^{*}$ for the dual of the Lie algebra $\mathfrak{g}$ corresponding to $G$, recall that if $H \in C^{\infty}\left(\mathfrak{g}^{*}\right)$ is a Hamiltonian of the dynamical system then the corresponding Hamiltonian equations, written with respect to the canonical linear Poisson structure $\{\cdot, \cdot\}_{\mathfrak{g}}$, take the following form

$$
\dot{\alpha}=-\operatorname{ad}_{d H_{\alpha}}^{\sharp}(\alpha),
$$

where $\alpha \in \mathfrak{g}^{*}$. In this description the Casimir functions provide only trivial first integrals with respect to the bracket $\{\cdot, \cdot\}_{\mathfrak{g}}$. The existence of a map $R \in \operatorname{End}(\mathfrak{g})$, which satisfies the so-called modified classical Yang-Baxter equation [17]

$$
[R(x), R(y)]-R([R(x), y]+[x, R(y)])=-[x, y]
$$


usually called an $R$-matrix, yields a new Lie bracket on $\mathfrak{g}$

$$
[x, y]_{R}:=\frac{1}{2}([R(x), y]+[x, R(y)]) .
$$

This Lie bracket defines the double Lie algebra $\overline{\mathfrak{g}}$, and the corresponding linear Poisson structure $\{\cdot, \cdot\}_{R}$ on $\mathfrak{g}^{*}$ associates to each Casimir function with respect to $\{\cdot, \cdot\}_{\mathfrak{g}}$, a non-trivial first integral of the original dynamical system. In this augmented setting the following holds:

(i) The Casimir functions corresponding to the original Poisson structure $\{\cdot, \cdot\}_{\mathfrak{g}}$ Poisson commute with respect to the new Poisson structure $\{\cdot, \cdot\}_{R}$.

(ii) For every Casimir function $H$, equation (1.1) written with respect to the Poisson structure $\{\cdot, \cdot\}_{R}$ assumes the form

$$
\dot{\alpha}=-\frac{1}{2} \operatorname{ad}_{R d H_{\alpha}}^{\sharp}(\alpha), \quad \forall \alpha \in \mathfrak{g}^{*} .
$$

Furthermore, under the assumption that $\mathfrak{g}$ is endowed with a non-degenerate, $\mathfrak{g}$-invariant bilinear form $(\cdot \mid \cdot)$, the previous equation can be written as the following Lie bracket flow equation

$$
\dot{x}_{\alpha}=\frac{1}{2}\left[x_{\alpha}, R d H_{\alpha}\right],
$$

where $x_{\alpha} \in \mathfrak{g}$ is the (unique) element such that $\left(x_{\alpha} \mid y\right)=\langle\alpha, y\rangle$, for all $y \in \mathfrak{g}$.

In many interesting cases $[1,8,11,19,22]$, the existence of such an $R$-matrix turns out to be equivalent to a decomposition of the Lie algebra $\mathfrak{g}=\mathfrak{g}_{+} \oplus \mathfrak{g}_{-}$, where $\mathfrak{g}_{ \pm}$are two Lie subalgebras of $\mathfrak{g}$. To any such decomposition corresponds a (local) decomposition of the corresponding Lie group $G \simeq G_{+} \times G_{-}$, where $\simeq$ in this case means a local diffeomorphism from a neighborhood of the identity $e \in G$ to a neighborhood of the identity $(e, e)$ of the (product) Lie group $G_{+} \times G_{-}$. Regarding equation (1.4), or equivalently equation (1.5), the following factorization theorem holds true. See the above references for details and background.

Theorem $1.1([17])$. Let $\alpha_{0} \in \mathfrak{g}^{*}$, let $H$ be a Casimir function of $\left(\mathfrak{g}^{*},\{\cdot, \cdot\}_{\mathfrak{g}}\right)$. Let $g_{ \pm}(t)$ be two smooth curves in $G$, such that:

(a) $g_{ \pm}(t) \in G_{ \pm}$for all $t$ for which they are defined,

(b) $g_{ \pm}(0)$ are both equal to the identity $e \in G$,

(c) they give a unique solution of the following factorization problem

$$
\exp \left(t d H_{\alpha_{0}}\right)=g_{+}(t) g_{-}(t)
$$

at least for $|t|<\epsilon$, with $\epsilon>0$.

Then the curve $t \rightsquigarrow \alpha(t), \alpha(0)=\alpha_{0}$, given by

$$
\alpha(t)=\operatorname{Ad}_{g_{+}^{-1}(t)}^{\sharp} \alpha_{0}=\operatorname{Ad}_{g_{-}(t)}^{\sharp} \alpha_{0}
$$

is a solution of equation (1.4).

The previous theorem connects a certain factorization of elements in the Lie group $G$ with the solution of Lie bracket flow equations in the corresponding (dual, $\mathfrak{g}^{*}$, of the) Lie algebra $\mathfrak{g}$. The main aim of this work is to explore this result in the framework of the Lie enveloping algebra of a post-Lie algebra defined on $\mathfrak{g}$ in terms of an $R$-matrix. 
A post-Lie algebra $[3,10,14,21]$ consists of a vector space $V$ equipped with two Lie brackets $[\cdot, \cdot]$ and $\llbracket \cdot, \cdot \rrbracket$ as well as a non-commutative and non-associative product $\triangleright: V \otimes V \rightarrow V$, such that the following identity holds

$$
\llbracket x, y \rrbracket=x \triangleright y-y \triangleright x+[x, y] .
$$

Further below we will state the precise definition and relations that characterize such an algebraic structure. In light of (1.2) and (1.3), the third product on the Lie algebra $(\mathfrak{g},[\cdot, \cdot])$ is given in terms of the $R$-matrix map [2], $x \triangleright y:=\left[\frac{1}{2}(R+\mathrm{id})(x), y\right], x, y \in \mathfrak{g}$. Lifting the post-Lie algebra to the Lie enveloping algebra of the Lie algebra $(\mathfrak{g},[\cdot, \cdot])$ allows us to define another associative product on $\mathcal{U}(\mathfrak{g})$, which is compatible with the latter's coalgebra structure [10]. The resulting Hopf algebra is isomorphic - as a Hopf algebra - to the Lie enveloping algebra of the second Lie algebra $(\overline{\mathfrak{g}}, \llbracket \cdot, \cdot \rrbracket)$. As a result $\mathcal{U}(\mathfrak{g})$ is equipped with two natural exponential maps, and the relation between those and the corresponding Lie groups in $\mathcal{U}(\mathfrak{g})$ is captured through a Magnustype differential equation. This gives rise to explicit solutions of the factorization (1.6) in Theorem 1.1.

We close this introduction with two remarks. First we would like to mention that differential geometry is a natural place to look for examples of post-Lie algebras. Indeed, a Koszul connection yields a $\mathbb{R}$-bilinear product on the space of smooth vector fields $\mathcal{X}(\mathcal{M})$ on a manifold $\mathcal{M}$. Flatness and constant torsion together with the Bianchi identities imply relation (1.7) between the Jacobi-Lie bracket of vector fields, the torsion itself, and the product defined in terms of the connection [14]. Second, we would like to stress that the formalism introduced in this note is based on the theory of classical $R$-matrices, and will be applied only in the context of classical dynamical systems, whose descriptions are given in terms of isospectral flows. However, saying this, it is worth mentioning that a similar formalism was used in [18] and [16] to the study of quantum groups and quantum integrable systems, see also Remark 4.12 below.

Outline of the paper. Sections 2 and 3 contain several preliminary results, which are crucial for the main statements of this paper, to be found in Sections 4 and 5. More precisely, in Section 2 we quickly recall the basic notions of $R$-matrices and their relations to factorization problems already mentioned above. Section 3 collects some basic facts about Lie-admissible algebras and post-Lie algebras. In particular, it is shown that solutions of the modified classical Yang-Baxter equation yield a post-Lie algebra structure on the original Lie algebra $\mathfrak{g}$. In Section 4, after recalling some important result on the universal enveloping algebra of a postLie algebra, we state a factorization theorem for the generators of a group $G^{*}$ sitting inside the universal enveloping algebra of any Lie algebra endowed with a solution of the modified classical Yang-Baxter equation. Furthermore, a distinguished linear isomorphism is defined between the universal enveloping algebra $\mathcal{U}(\mathfrak{g})$ of a Lie algebra $\mathfrak{g}$ supporting an $R$-matrix, and the universal enveloping algebra $\mathcal{U}(\overline{\mathfrak{g}})$ of the corresponding double Lie algebra. Moreover, it is shown that by swapping the associative product of $\mathcal{U}(\mathfrak{g})$ for a new product defined by extending the post-Lie product defined on $\mathfrak{g}$ in terms of the $R$-matrix to $\mathcal{U}(\mathfrak{g})$, such a linear isomorphism becomes a morphism of associative algebras. Finally, in Section 5 the post-Lie algebra structure is invoked to show how the BCH-recursion follows as the solution of a Magnus-type differential equation. This is then applied to Lie bracket flows. It is shown that the solution of a Lie bracket flow on a Lie algebra $\mathfrak{g}$ endowed with a solution of the modified classical Yang-Baxter equation can be described, under suitable convergence assumptions, in terms of the generators of the group $G^{*}$.

In the following the ground field $\mathbb{K}$ is of characteristic zero, and $\mathbb{K}$-algebras are assumed to be associative and unital, if not stated otherwise. 


\section{$2 \quad \boldsymbol{R}$-matrices and factorization}

We consider a Lie algebra $\mathfrak{g}$ together with an $R$-matrix $\pi_{+}: \mathfrak{g} \rightarrow \mathfrak{g}$. Both $\pi_{+}$and the map $\pi_{-}:=\mathrm{id}-\pi_{+}$satisfy the Lie algebra identity

$$
\left[\pi_{ \pm}(x), \pi_{ \pm}(y)\right]+\pi_{ \pm}([x, y])=\pi_{ \pm}\left(\left[\pi_{ \pm}(x), y\right]+\left[x, \pi_{ \pm}(y)\right]\right) .
$$

Note that the map $R:=\mathrm{id}-2 \pi_{+}$satisfies the modified classical Yang-Baxter equation (1.2). For details we refer the reader to $[1,11,16,17]$. In the following theorem we collect some well-known results.

Theorem 2.1 ([17]). If $\pi_{-}$is a solution of $(2.1)$, then the bracket $\llbracket \cdot, \cdot \rrbracket$ defined for all $x, y \in \mathfrak{g}$ by

$$
\llbracket x, y \rrbracket:=\left[\pi_{-}(x), y\right]+\left[x, \pi_{-}(y)\right]-[x, y]=\left[\pi_{-}(x), \pi_{-}(y)\right]-\left[\pi_{+}(x), \pi_{+}(y)\right],
$$

satisfies the Jacobi identity, and therefore defines the so-called double Lie algebra $\overline{\mathfrak{g}}$ on the vector space underlying $\mathfrak{g}$. Both images $\mathfrak{g}_{ \pm}:=\pi_{ \pm}(\mathfrak{g})$ form Lie subalgebras, and $\pi_{\mp}(\llbracket x, y \rrbracket)=$ $\pm\left[\pi_{\mp}(x), \pi_{\mp}(y)\right]$.

As mentioned in the introduction, solutions of (2.1) are intimately related to factorizations of the Lie group $G$, see [11] for details. For simplicity we assume that $\pi_{+}$is a projector - which is covering many interesting cases. The subgroups corresponding to the Lie subalgebras $\mathfrak{g}_{ \pm}$are denoted $G_{ \pm}$. For $a_{0} \in \mathfrak{g}$ and a small enough $t$, that is, in a sufficiently small neighborhood of the unit of the group $G$ corresponding to $\mathfrak{g}$, the following unique factorization holds

$$
\exp \left(t a_{0}\right)=g_{+}(t) g_{-}(t),
$$

with $g_{ \pm}(t) \in G_{ \pm}$for all $t$ for which they are defined. Define the map

$$
a(t):=g_{+}^{-1}(t) a_{0} g_{+}(t)=g_{-}(t) a_{0} g_{-}^{-1}(t),
$$

and recall that it satisfies the Lie bracket initial value problem

$$
\dot{a}(t)=\left[a(t), p_{+}(t)\right], \quad a(0)=a_{0},
$$

with $p_{+}(t):=g_{+}^{-1}(t) \frac{d}{d t}\left(g_{+}(t)\right)$. From (2.3) it follows that

$$
a(t)=g_{+}^{-1}(t) \frac{d}{d t}\left(g_{+}(t)\right)+\frac{d}{d t}\left(g_{-}(t)\right) g_{-}^{-1}(t) .
$$

Since $\pi_{ \pm}$are projectors we obtain $g_{+}^{-1}(t) \frac{d}{d t}\left(g_{+}(t)\right) \in \mathfrak{g}_{+}$and $\frac{d}{d t}\left(g_{-}(t)\right) g_{-}^{-1}(t) \in \mathfrak{g}_{-}$, and therefore $p_{+}(t)=\pi_{+}(a(t))$.

Anticipating what follows below, we remark that in [6] the function $\Omega\left(t ; a_{0}\right)$ was described in terms of a Magnus-type differential equation, such that $g_{+}(t)=\exp \left(\Omega\left(t ; a_{0}\right)\right)$. In this paper we will describe this Magnus-type differential equation using a post-Lie algebra, and thereby clarify its link to (2.3) by showing that its solution is given in terms of the BCH-recursion [9].

\section{$3 \quad$ Lie-admissible algebras, post-Lie algebras and $R$-matrices}

Definition 3.1. A $\mathbb{K}$-algebra $(A, \cdot)$, not necessarily associative, is called Lie-admissible if the commutator $[a, b]:=a \cdot b-b \cdot a$ defines a Lie bracket. In this case, the corresponding Lie algebra $(A,[\cdot, \cdot])$ will be denoted by $A_{\text {Lie }}$.

Note that associative $\mathbb{K}$-algebras are Lie-admissible. Another class of Lie-admissible algebras is introduced in the following definition. 
Definition 3.2. The algebra $(A, \diamond)$ with binary product $\diamond: A \otimes A \rightarrow A$ will be called a (left) pre-Lie algebra, if for all $x, y, z \in A$

$$
\mathrm{a}_{\diamond}(x, y, z)=\mathrm{a}_{\diamond}(y, x, z)
$$

where $\mathrm{a}_{\diamond}(x, y, z):=x \diamond(y \diamond z)-(x \diamond y) \diamond z$ is the associator.

Pre-Lie algebras are Lie-admissible. Indeed, note that identity (3.1) can be written as $\ell_{[x, y] \diamond}(z)=\left[\ell_{x \diamond}, \ell_{y \diamond}\right](z)$, where the linear map $\ell_{x \diamond}: A \rightarrow A$ is defined by $\ell_{x \diamond}(y):=x \diamond y$ and the bracket on the left-hand side is defined by $[x, y]:=x \diamond y-y \diamond x$. As a consequence it satisfies the Jacobi identity, turning $A$ into a Lie algebra. See $[4,13]$ for more details. We now turn to the definition of post-Lie algebra following reference [14].

Definition 3.3. Let $(\mathfrak{g},[\cdot, \cdot])$ be a Lie algebra, and let $\triangleright: \mathfrak{g} \otimes \mathfrak{g} \rightarrow \mathfrak{g}$ be a binary product such that, for all $x, y, z \in \mathfrak{g}$,

$$
x \triangleright[y, z]=[x \triangleright y, z]+[y, x \triangleright z],
$$

and

$$
[x, y] \triangleright z=\mathrm{a}_{\triangleright}(x, y, z)-\mathrm{a}_{\triangleright}(y, x, z) .
$$

Then the triplet $(\mathfrak{g},[\cdot, \cdot], \triangleright)$ is called a post-Lie algebra.

Remark 3.4. Let $(\mathfrak{g},[\cdot, \cdot], \triangleright)$ be a post-Lie algebra.

a) If $x \triangleright y:=x \triangleright y+[x, y]$, then $(\mathfrak{g},-[\cdot, \cdot], \triangleright)$ is a post-Lie algebra.

b) If $x \succ y:=x \triangleright y+\frac{1}{2}[x, y]$, then $(\mathfrak{g}, \succ)$ is Lie-admissible.

Proposition 3.5. Let $(\mathfrak{g},[\cdot, \cdot], \triangleright)$ be a post-Lie algebra. The bracket

$$
\llbracket x, y \rrbracket:=x \triangleright y-y \triangleright x+[x, y]
$$

satisfies the Jacobi identity for all $x, y \in \mathfrak{g}$. The Lie algebra is written as $(\overline{\mathfrak{g}}, \llbracket \cdot, \cdot \rrbracket)$.

Remark 3.6. Pre- and post-Lie algebras are important in the theory of numerical methods for differential equations. We refer the reader to $[4,7,10,13,14]$ for background and details.

In the introduction we pointed at an archetypal example of post-Lie algebra coming from differential geometry. Here we will present an algebraic example using $R$-matrices. Let $\pi_{+} \in$ $\operatorname{End}(\mathfrak{g})$ satisfy identity $(2.1)$, and define the following binary product on $\mathfrak{g}$ :

$$
a \triangleright b:=-\left[\pi_{+}(a), b\right] .
$$

Theorem 3.7 ([2]). The product (3.3) defines a post-Lie algebra structure on $\mathfrak{g}$.

It turns out that the new Lie bracket defined in terms of this post-Lie product and the original Lie bracket is the one given in (2.2). Indeed, for $\pi_{+}:=\mathrm{id}-\pi_{-}$,

$$
x \triangleright y-y \triangleright x+[x, y]=\left[\pi_{-}(x), y\right]+\left[x, \pi_{-}(y)\right]-[x, y]=\llbracket x, y \rrbracket .
$$

Remark 3.8. Returning to item a) in Remark 3.4, note that the second post-Lie algebra $(\mathfrak{g},-[\cdot, \cdot], \boldsymbol{})$ is defined in terms of $\pi_{-}:=\mathrm{id}-\pi_{+}:$the product is $x \triangleright y:=x \triangleright y+[x, y]=\left[\pi_{-}(a), b\right]$.

As for item $\mathrm{b}$ ), one finds that the Lie-admissible algebra $(\mathfrak{g}, \succ)$ is defined through the binary composition $x \succ y:=x \triangleright y+\frac{1}{2}[x, y]=\left[\frac{1}{2} R(x), y\right]$, where $R:=\mathrm{id}-2 \pi_{-}$. The Lie bracket (3.2) is then given by $\llbracket x, y \rrbracket:=x \succ y-y \succ x$, which is just another way of writing (1.3). With $\tilde{R}:=\frac{1}{2} R$, one can deduce from $[\tilde{R}(x), \tilde{R}(y)]-\tilde{R}([\tilde{R}(x), y]+[x, \tilde{R}(y)])=-\frac{1}{4}[x, y]$ that

$$
\mathrm{a}_{\succ}(x, y, z)-\mathrm{a}_{\succ}(y, x, z)=-\frac{1}{4}[[x, y], z] .
$$




\section{Lie enveloping algebra of a post-Lie algebra}

In [10] the Lie enveloping algebra of a post-Lie algebra was described. Here we recall the basic results without proofs. Let $(\mathfrak{g},[\cdot, \cdot], \triangleright)$ be a post-Lie algebra, and $\mathcal{U}(\mathfrak{g})$ the universal enveloping algebra of the Lie algebra $(\mathfrak{g},[\cdot, \cdot])$. Recall that $\mathcal{U}(\mathfrak{g})$ with concatenation product is a noncommutative, cocommutative filtered Hopf algebra generated by $\mathfrak{g} \hookrightarrow \mathcal{U}(\mathfrak{g})$. The coshuffle coproduct is defined for $x \in \mathfrak{g}$ by $\Delta(x):=x \otimes \mathbf{1}+\mathbf{1} \otimes x$, i.e., elements of $\mathfrak{g}$ are primitive. It is extended multiplicatively to all of $\mathcal{U}(\mathfrak{g})$. We use Sweedler's notation for the coproduct: $\Delta(T)=$ : $T_{(1)} \otimes T_{(2)}$, see [20]. The counit is denoted by $\epsilon: \mathcal{U}(\mathfrak{g}) \rightarrow \mathbb{K}$, and the antipode $S: \mathcal{U}(\mathfrak{g}) \rightarrow \mathcal{U}(\mathfrak{g})$ is defined through $S\left(x_{1} \cdots x_{k}\right):=(-1)^{k} x_{k} \cdots x_{1}$ (in particular $S(x)=-x$ for all $x \in \mathfrak{g}$ ). Finally, remember that the universal property of $\mathcal{U}(\mathfrak{g})$ implies that if $A$ is an associative algebra and $f: \mathfrak{g} \rightarrow A_{\text {Lie }}$ is a homomorphism of Lie algebras, then there exists a unique (unital associative algebra) morphism $F: \mathcal{U}(\mathfrak{g}) \rightarrow A$ such that $F \circ i=f$, where $i: \mathfrak{g} \rightarrow \mathcal{U}(\mathfrak{g})$ is the canonical embedding.

We have seen in Proposition 3.5 that the vector space underlying a post-Lie algebra carries two Lie algebras, $(\mathfrak{g},[\cdot, \cdot])$ and $(\overline{\mathfrak{g}}, \llbracket \cdot, \cdot \rrbracket)$, related via the post-Lie product

$$
\llbracket x, y \rrbracket=x \triangleright y-y \triangleright x+[x, y] .
$$

In what follows, $(\mathcal{U}(\overline{\mathfrak{g}}), \cdot)$ will denote the universal enveloping algebra of the Lie algebra $(\overline{\mathfrak{g}}, \llbracket \cdot, \cdot \rrbracket)$. In the next proposition the post-Lie product is extended to $\mathcal{U}(\mathfrak{g})$.

Theorem 4.1 ([10]). There is a unique extension of the post-Lie product $\triangleright$ from $\mathfrak{g}$ to $\mathcal{U}(\mathfrak{g})$. On $(\mathcal{U}(\mathfrak{g}), \triangleright)$ the product

$$
A * B:=A_{(1)}\left(A_{(2)} \triangleright B\right)
$$

for $A, B \in \mathcal{U}(\mathfrak{g})$ is associative and unital. Moreover, $(\mathcal{U}(\mathfrak{g}), *, \Delta)$ is a Hopf algebra isomorphic to $(\mathcal{U}(\overline{\mathfrak{g}}), \cdot, \Delta)$.

Remark 4.2. A few remarks are in order.

i) The last statement in Theorem 4.1 appeared in the context of pre-Lie algebras in [15], and we refer the reader to [10] for details. For the notation, see item (ii) below.

ii) In what follows we will be working with three Hopf algebras. We will consider $(\mathcal{U}(\mathfrak{g}), \cdot, \Delta)$, i.e., the universal enveloping algebra of $\mathfrak{g}$, and the Hopf algebra $(\mathcal{U}(\mathfrak{g}), *, \Delta)$, both defined on the same underlying vector space, whose products are $\mu .(x, y)=x \cdot y$ and $\mu_{*}(x, y)=x * y$, respectively. Note that the coproduct $\Delta$ is the same for both, given by the one originally defined on the universal enveloping algebra $(\mathcal{U}(\mathfrak{g}), \cdot, \Delta)$. According to the last statement in Theorem 4.1 this map is an algebra morphism on $(\mathcal{U}(\mathfrak{g}), *)$. The third Hopf algebra we will consider is the universal enveloping algebra of the Lie algebra $(\overline{\mathfrak{g}}, \llbracket \cdot, \cdot \rrbracket)$. Even though this Hopf algebra should be denoted as $\left(\mathcal{U}(\overline{\mathfrak{g}}), \overline{-}^{-} \Delta_{\text {-) }}\right)$ we stick to a simplified notation, and denote its product and coproduct by $\cdot$ and $\Delta$, respectively.

iii) From now on, suitable completions of the above Hopf algebras will be considered, still denoted by the same symbols $\mathcal{U}(\mathfrak{g}):=(\mathcal{U}(\mathfrak{g}), \cdot, \Delta),, \mathcal{U}_{*}(\mathfrak{g}):=(\mathcal{U}(\mathfrak{g}), *, \Delta)$ and $\mathcal{U}(\overline{\mathfrak{g}}):=$ $(\mathcal{U}(\overline{\mathfrak{g}}), \cdot, \Delta)$. Then, for any element $v \in \mathfrak{g}$, one may consider the elements exp $v$, $\exp ^{\bar{v}} v$ and $\exp ^{*} v$. For example, with $v^{* n}$ denoting the $n$-fold product $v * \cdots * v$,

$$
\exp ^{*} v:=\sum_{n \geq 0} \frac{v^{* n}}{n !} \in \mathcal{U}_{*}(\mathfrak{g}) .
$$

A simple computation shows that each of these elements is group-like in the corresponding Hopf algebra. For this reason one may consider $G, G^{*}$ and $\bar{G}$, the groups generated for $v \in \mathfrak{g}$ 
by the products of the elements of type $\exp ^{*} v, \exp ^{*} v$ and $\exp ^{\top} v$, respectively. Moreover, to simplify notation, we will write $\exp v$ and $\exp v$ to denote the exponentials of $v$ in $\mathcal{U}(\mathfrak{g})$ and $\mathcal{U}(\overline{\mathfrak{g}})$, respectively.

iv) In what follows we will often need to use the classical BCH-formula. Recall that $\mathrm{BCH}: \mathfrak{g} \times$ $\mathfrak{g} \rightarrow \mathfrak{g}$ is defined such that $\exp x \exp y=\exp \operatorname{BCH}(x, y)$, and

$$
\operatorname{BCH}(x, y)=x+y+\frac{1}{2}[x, y]+\frac{1}{12}[x,[x, y]]+\frac{1}{12}[y,[y, x]]-\frac{1}{24}[y,[x,[x, y]]]+\cdots,
$$

where $x, y \in \mathfrak{g}$. The reduced $\mathrm{BCH}$-formula is defined by $\overline{\mathrm{BCH}}(x, y):=\mathrm{BCH}(x, y)-x-y$. From a formal point of view the BCH-formula maps elements from $\mathfrak{g}$ into the completion of $\mathcal{U}(\mathfrak{g})$. Without further comments, we will therefore assume that it is convergent. However, when working locally we will restrict ourselves to a suitable neighborhood $U$ of the zero element of the Lie algebra $\mathfrak{g}$.

Beside the exponential maps introduced in (iii), we need the following ordered post-Lie exponential in $\mathcal{U}(\mathfrak{g})$.

Definition 4.3. For any primitive element $a \in \mathfrak{g}$, the right-ordered exponential $\exp ^{\triangleright}: \mathfrak{g} \rightarrow \mathcal{U}(\mathfrak{g})$ is defined for $b \in \mathcal{U}(\mathfrak{g})$ as

$$
\exp ^{\triangleright}(a) b:=b+a \triangleright b+\frac{1}{2 !} a \triangleright(a \triangleright b)+\frac{1}{3 !} a \triangleright(a \triangleright(a \triangleright b))+\cdots .
$$

From the identity $A \triangleright(B \triangleright C)=\left(A_{(1)}\left(A_{(2)} \triangleright B\right)\right) \triangleright C$, which holds for all $A, B, C \in \mathcal{U}(\mathfrak{g})$, see [10], it follows immediately that in $\mathcal{U}(\mathfrak{g})$ :

$$
\exp ^{\triangleright}(a) b=\exp ^{*}(a) \triangleright b .
$$

We now consider these results in the context of a post-Lie algebra which is defined in terms of an $R$-matrix $\pi_{+} \in \operatorname{End}(\mathfrak{g})$ satisfying identity (2.1). First recall that any element $v \in \mathfrak{g}$ may be decomposed: $v=\pi_{-}(v)+\pi_{+}(v)=: v_{-}+v_{+}$, and let $(\mathfrak{g},[\cdot, \cdot], \triangleright)$ be the corresponding post-Lie algebra with $a \triangleright b:=-\left[\pi_{+}(a), b\right]$. Then:

Proposition 4.4. For every $v, w \in \mathfrak{g}$ the following equality

$$
\exp ^{*}(v) \triangleright w=\exp \left(-\pi_{+}(v)\right) w \exp \left(\pi_{+}(v)\right)=\exp \left(-\operatorname{ad}_{\pi_{+}(v)}\right) w
$$

holds in $\mathcal{U}(\mathfrak{g})$.

Proof. Note that this result implies that $\exp ^{*}(v) \triangleright w \in \mathfrak{g}$ for $v, w \in \mathfrak{g}$.

$$
\begin{aligned}
\exp ^{\triangleright}(v) w & =w+v \triangleright w+\frac{1}{2} v \triangleright(v \triangleright w)+\cdots=w-\left[\pi_{+}(v), w\right]+\frac{1}{2}\left[\pi_{+}(v),\left[\pi_{+}(v), w\right]\right]-\cdots \\
& =\exp \left(-\pi_{+}(v)\right) w \exp \left(\pi_{+}(v)\right) .
\end{aligned}
$$

The next proposition is a natural factorization statement for the generators of the group $G^{*} \subset \mathcal{U}(\mathfrak{g})$, see item (iii) in Remark 4.2.

Proposition 4.5. For each $v \in \mathfrak{g}$ the following factorization holds:

$$
\exp ^{*}(v)=\exp \left(v_{-}\right) \exp \left(v_{+}\right)
$$


Proof. Recall that for $v \in \mathfrak{g} \hookrightarrow \mathcal{U}(\mathfrak{g})$, one has $v \triangleright v=-\left[v_{+}, v_{-}\right]$, where $v_{ \pm}:=\pi_{ \pm}(v)$, and $v \triangleright v_{+}=0$. Next we show inductively that $\frac{1}{n !} v^{* n}=\sum_{k+l=n} \frac{1}{k !} \frac{1}{l !} v_{-}^{k} v_{+}^{l}$. For $n=2$ one finds that:

$$
\frac{1}{2} v * v=\frac{1}{2} v v+\frac{1}{2} v \triangleright v=\frac{1}{2} v v-\frac{1}{2}\left[v_{+}, v_{-}\right]=\frac{1}{2} v_{+} v_{+}+\frac{1}{2} v_{-} v_{-}+v_{-} v_{+} .
$$

For $n>2$ we have

$$
\begin{aligned}
\frac{1}{n !} v^{* n}= & \frac{1}{n(n-1) !} v * v^{* n-1}=\frac{1}{n} v * \sum_{k=0}^{n-1} \frac{1}{k !} \frac{1}{(n-1-k) !} v_{-}^{k} v_{+}^{n-1-k} \\
= & \frac{1}{n}\left(\sum_{k=0}^{n-1} \frac{1}{k !} \frac{1}{(n-1-k) !} v v_{-}^{k} v_{+}^{n-1-k}+\sum_{k=0}^{n-1} \frac{1}{k !} \frac{1}{(n-1-k) !} v \triangleright\left(v_{-}^{k} v_{+}^{n-1-k}\right)\right) \\
= & \frac{1}{n}\left(\sum_{k=0}^{n-1} \frac{1}{k !} \frac{1}{(n-1-k) !}\left(v_{-}^{k+1} v_{+}^{n-1-k}+v_{+} v_{-}^{k} v_{+}^{n-1-k}\right)\right. \\
& \left.+\sum_{k=1}^{n-1} \frac{1}{k !} \frac{1}{(n-1-k) !}\left(v \triangleright v_{-}^{k}\right) v_{+}^{n-1-k}\right),
\end{aligned}
$$

where we used that $v \triangleright v_{+}=0$. Furthermore

$$
\begin{aligned}
\left(v \triangleright v_{-}^{k}\right) & =\left(v \triangleright v_{-}\right) v_{-}^{k-1}+\sum_{m=1}^{k-1} v_{-}^{m}\left(v \triangleright v_{-}\right) v_{-}^{k-m-1} \\
& =-\left[v_{+}, v_{-}\right] v_{-}^{k-1}-\sum_{m=1}^{k-1} v_{-}^{m}\left(\left[v_{+}, v_{-}\right]\right) v_{-}^{k-m-1} \\
& =-v_{+} v_{-}^{k}+v_{-} v_{+} v_{-}^{k-1}-v_{-} v_{+} v_{-}^{k-1}+v_{-}^{2} v_{+} v_{-}^{k-2}-\sum_{m=2}^{k-1} v_{-}^{m}\left(\left[v_{+}, v_{-}\right]\right) v_{-}^{k-m-1} \\
& =-v_{+} v_{-}^{k}+v_{-}^{k} v_{+},
\end{aligned}
$$

which implies that

$$
\begin{aligned}
\frac{1}{n !} v^{* n}= & \frac{1}{n}\left(\sum_{k=0}^{n-1} \frac{1}{k !} \frac{1}{(n-1-k) !}\left(v_{-}^{k+1} v_{+}^{n-1-k}+v_{+} v_{-}^{k} v_{+}^{n-1-k}\right)\right. \\
& \left.+\sum_{k=0}^{n-1} \frac{1}{k !} \frac{1}{(n-1-k) !}\left(-v_{+} v_{-}^{k}+v_{-}^{k} v_{+}\right) v_{+}^{n-1-k}\right) \\
= & \frac{1}{n}\left(\sum_{k=1}^{n} \frac{1}{(k-1) !} \frac{1}{(n-k) !} v_{-}^{k} v_{+}^{n-k}+\sum_{k=0}^{n-1} \frac{1}{k !} \frac{1}{(n-1-k) !} v_{-}^{k} v_{+}^{n-k}\right) \\
= & \frac{1}{n !} v_{-}^{n}+\frac{1}{n !} v_{+}^{n}+\frac{1}{n}\left(\sum_{k=1}^{n-1} \frac{1}{(k-1) !} \frac{1}{(n-k) !} v_{-}^{k} v_{+}^{n-k}+\sum_{k=1}^{n-1} \frac{1}{k !} \frac{1}{(n-1-k) !} v_{-}^{k} v_{+}^{n-k}\right) \\
= & \frac{1}{n !} v_{-}^{n}+\frac{1}{n !} v_{+}^{n}+\sum_{k=1}^{n-1} \frac{1}{k !} \frac{1}{(n-k) !} v_{-}^{k} v_{+}^{n-k} .
\end{aligned}
$$

\section{Remark 4.6.}

a) Another way to prove equality (4.2) is to show that both sides solve the same initial value problem. For this we take a local point of view by assuming a small enough $t$ such that 
$\exp ^{*}(t v)$ and $\exp \left(t v_{-}\right) \exp \left(t v_{+}\right)$both lie in a sufficiently small neighborhood of the unit of the Lie group $G$ corresponding to the Lie algebra $\mathfrak{g}$. Then $y_{1}(t):=\exp ^{*}(t v)$ and $y_{2}(t):=$ $\exp \left(t v_{-}\right) \exp \left(t v_{+}\right)$are solutions of

$$
\dot{y}(t)=y(t)\left(\exp \left(-\pi_{+}(t v)\right) v \exp \left(\pi_{+}(t v)\right)\right), \quad y(0)=1 .
$$

Indeed,

$$
\begin{aligned}
\dot{y}_{1}(t) & =y_{1}(t) * v=y_{1}(t)\left(y_{1}(t) \triangleright v\right)=y_{1}(t)\left(\exp ^{*}(t v) \triangleright v\right) \\
& =y_{1}(t)\left(\exp \left(-\pi_{+}(t v)\right) v \exp \left(\pi_{+}(t v)\right)\right)
\end{aligned}
$$

and

$$
\begin{aligned}
\dot{y}_{2}(t) & =\exp \left(t v_{-}\right) v_{-} \exp \left(t v_{+}\right)+\exp \left(t v_{-}\right) v_{+} \exp \left(t v_{+}\right) \\
& =\exp \left(t v_{-}\right) v \exp \left(t v_{+}\right)=y_{2}(t)\left(\exp \left(-\pi_{+}(t v)\right) v \exp \left(\pi_{+}(t v)\right)\right) .
\end{aligned}
$$

From which the statement follows by uniqueness of the solution.

b) Returning to item (iv) of Remark 4.2, we find that (4.2) implies

$$
\exp ^{*}(v)=\exp \left(v_{-}\right) \exp \left(v_{+}\right)=\exp \left(\operatorname{BCH}\left(v_{-}, v_{+}\right)\right)
$$

In light of Proposition 3.11 in [10], which says that for $v \in \mathfrak{g}$ and sufficiently small enough $t$

$$
\exp ^{*}(t v)=\exp (\theta(t v))
$$

where $\dot{\theta}(t v)=\operatorname{dexp}_{\theta(t v)}^{-1}(\exp (\theta(t v)) \triangleright v)$, we see that $\theta(t v)=\mathrm{BCH}\left(t v_{-}, t v_{+}\right)$.

If the $R$-matrix corresponds to a Lie algebra that splits into a direct sum of vector subspaces, $\mathfrak{g}=\mathfrak{g}_{+} \oplus \mathfrak{g}_{-}$, then in addition to $v \triangleright v_{+}=0$, we have that $v_{-} \triangleright v_{-}=v_{+} \triangleright v_{+}=0$. This implies the next result.

Corollary 4.7. In the case of an R-matrix $\pi_{+}$which is a projector on $\mathfrak{g}$, we find that

$$
\exp ^{*}\left(v_{ \pm}\right)=\exp \left(v_{ \pm}\right)
$$

The result in Proposition 4.5 has a Hopf algebraic formulation. Recall that the universal property of $\mathcal{U}(\overline{\mathfrak{g}})$ implies that the $R$-matrices $\mathrm{r}_{+}:=-\pi_{+}$and $\mathrm{r}_{-}:=\pi_{-}$become unital algebra morphism from $\mathcal{U}(\overline{\mathfrak{g}})$ to $\mathcal{U}(\mathfrak{g})$.

Definition 4.8. Let $F: \mathcal{U}(\overline{\mathfrak{g}}) \rightarrow \mathcal{U}(\mathfrak{g})$ be the map defined by

$$
F:=\mu \circ(\mathrm{id} \otimes S) \circ\left(\mathrm{r}_{-} \otimes \mathrm{r}_{+}\right) \circ \Delta,
$$

where $\mu$ denotes the product in $\mathcal{U}(\mathfrak{g})$.

Remark 4.9. For $X \in \mathcal{U}(\mathfrak{g})$, using the Sweedler notation, one can write

$$
F(X)=(-1)^{\left|X_{(2)}\right|} X_{-(1)} S\left(X_{+(2)}\right),
$$

where $|X|$ denotes the degree of a homogenous $X \in \mathcal{U}(\mathfrak{g})$.

We can now prove the following result.

Proposition 4.10. The map $F$ is an algebra morphism from $\mathcal{U}(\overline{\mathfrak{g}})$ to $\mathcal{U}_{*}(\mathfrak{g})$, i.e., it is a linear map such that:

$$
F\left(x_{1} \cdots x_{n}\right)=F\left(x_{1}\right) * \cdots * F\left(x_{n}\right),
$$

for all monomials $x_{1} \cdots x_{n} \in \mathcal{U}(\overline{\mathfrak{g}})$. 
Proof. First observe that since every $x \in \overline{\mathfrak{g}}$ is a primitive element in $\mathcal{U}(\overline{\mathfrak{g}})$,

$$
F(x)=\mathrm{r}_{-}(x)-\mathrm{r}_{+}(x)=x_{-}+x_{+}=x .
$$

Then note that for every $x, y \in \mathfrak{g}$ one finds:

$$
F(x \cdot y)=x_{-} y_{-}+y_{+} x_{+}+x_{-} y_{+}+y_{-} x_{+}=x y-\left[\pi_{+}(x), y\right]=x * y=F(x) * F(y) .
$$

Let $X:=x_{1} \cdot x_{2} \cdots x_{n}=x_{1} \cdot X^{\prime} \in \mathcal{U}(\overline{\mathfrak{g}}), x_{i} \in \mathfrak{g}$, and calculate

$$
\begin{aligned}
F(X)= & \mu \circ(\mathrm{id} \otimes S) \circ\left(\mathrm{r}_{-} \otimes \mathrm{r}_{+}\right) \circ \Delta\left(x_{1} \cdot X^{\prime}\right) \\
= & \mu \circ(\mathrm{id} \otimes S) \circ\left(\mathrm{r}_{-} \otimes \mathrm{r}_{+}\right) \circ \Delta\left(x_{1}\right) \cdot \Delta .\left(X^{\prime}\right) \\
= & \mu \circ\left(x_{1-} \otimes \mathbf{1}\right)(\mathrm{id} \otimes S) \circ\left(\mathrm{r}_{-} \otimes \mathrm{r}_{+}\right) \circ \Delta\left(X^{\prime}\right) \\
& -\mu \circ(\mathrm{id} \otimes S) \circ\left(\mathbf{1} \otimes x_{1+}\right)\left(\mathrm{r}_{-} \otimes \mathrm{r}_{+}\right) \circ \Delta\left(X^{\prime}\right) \\
= & \mu \circ\left(x_{1-} \otimes \mathbf{1}\right)(\mathrm{id} \otimes S) \circ\left(\mathrm{r}_{-} \otimes \mathrm{r}_{+}\right) \circ \Delta\left(X^{\prime}\right) \\
& +\mu \circ\left(\left((\mathrm{id} \otimes S) \circ\left(\mathrm{r}_{-} \otimes \mathrm{r}_{+}\right) \circ \Delta\left(X^{\prime}\right)\right)\left(\mathbf{1} \otimes x_{1+}\right)\right) \\
= & x_{1-}\left(x_{2} * \cdots * x_{n}\right)+\left(x_{2} * \cdots * x_{n}\right) x_{1+} \\
= & x_{1}\left(x_{2} * \cdots * x_{n}\right)-x_{1+}\left(x_{2} * \cdots * x_{n}\right)+\left(x_{2} * \cdots * x_{n}\right) x_{1+} \\
= & x_{1} * x_{2} * \cdots * x_{n}=F\left(x_{1}\right) * F\left(x_{2}\right) * \cdots * F\left(x_{n}\right) .
\end{aligned}
$$

We conclude this section by observing that using the map $F$ of Definition 4.8, one can recover the factorization described in Proposition 4.5.

Corollary 4.11. For every $v \in \mathfrak{g}$,

$$
F(\exp (v))=\exp \left(v_{-}\right) \exp \left(v_{+}\right)=\exp ^{*}(v) .
$$

Proof. The result follows from the definition of the map $F$ and from the property of $\exp ^{*}(v)$ being a group-like element in $\mathcal{U}(\overline{\mathfrak{g}})$.

Remark 4.12. We compare the map $F$ defined in (4.3) with formula (1.19) in [16]. The authors of [16] work with a factorizable $r$-matrix, i.e., with an element $r \in \mathfrak{g} \otimes \mathfrak{g}$, satisfying the classical Yang-Baxter equation, and having a symmetric part defining a linear isomorphism $I: \mathfrak{g}^{*} \rightarrow \mathfrak{g}$. The element $r$ permits one to define a Lie algebra struture on the dual vector space $\mathfrak{g}^{*}$. Let $\mathcal{U}\left(\mathfrak{g}^{*}\right)$ be the corresponding universal enveloping algebra. Then in [16] it is proven that the map

$$
\mathcal{I}=\mu \circ(\mathrm{id} \otimes S) \circ\left(r_{+} \otimes r_{-}\right) \circ \Delta: \mathcal{U}\left(\mathfrak{g}^{*}\right) \rightarrow \mathcal{U}(\mathfrak{g})
$$

is a linear isomorphism extending $I$.

It is easy to show that the Hopf algebra $\mathcal{U}\left(\mathfrak{g}^{*}\right)$ is isomorphic to $\mathcal{U}(\overline{\mathfrak{g}})$, where the $R$-matrix defining the Lie algebra structure in $\overline{\mathfrak{g}}$ is obtained from $r$ and $I$ as $R:=\underline{r} \circ I$. Here $\underline{r}: \mathfrak{g}^{*} \rightarrow \mathfrak{g}$ is defined by $\langle\beta, \underline{r}(\alpha)\rangle=\langle\alpha \otimes \beta, r\rangle$, for all $\alpha, \beta \in \mathfrak{g}^{*}$. After identifying $\mathcal{U}\left(\mathfrak{g}^{*}\right)$ with $\mathcal{U}(\overline{\mathfrak{g}})$ using this isomorphism, the map $\mathcal{I}$ becomes the map $F$, seen as a linear map between $\mathcal{U}(\overline{\mathfrak{g}})$ and $\mathcal{U}(\mathfrak{g})$. Proposition 4.10 states that, at the cost of trading the associative product of $\mathcal{U}(\mathfrak{g})$ for the product $*$ defined in (4.1), $F$ becomes an isomorphism of associative algebras.

It is also worth mentioning that in [18], the (inverse) of the linear isomorphism defined in formula (4.3) was shown to restrict to an algebra homomorphism between the center of $\mathcal{U}(\mathfrak{g})$ and $\mathcal{U}(\overline{\mathfrak{g}})$. This homomorphism was then used in the same paper to define a quantization of the symmetric algebra $\mathcal{S}(\overline{\mathfrak{g}})$, i.e., a linear map $q: \mathcal{S}(\overline{\mathfrak{g}}) \rightarrow \mathcal{U}(\overline{\mathfrak{g}})$, compatible with the filtrations and preserving the so called total symbol of the elements of $\mathcal{U}(\overline{\mathfrak{g}})$, see $[18, \mathrm{p} .3414]$. The quantization of $\mathcal{S}(\overline{\mathfrak{g}})$ so obtained was then used to construct the quantum integral of motions for systems with linear Poisson brackets.

Regarding the aforementioned remark, we should emphasize that in this note our main interest lies in applying Magnus type formulas combined with post-Lie structures to solve classical Lax type equations, we will refrain from further commenting on possible applications of the formalism here introduced in the context of the quantum dynamical systems. 


\section{Post-Lie algebra and Lie-bracket flows}

In this section the assumptions and notations made explicit in items (ii) and (iii) of Remark 4.2 apply ${ }^{1}$. We start by recalling the so-called BCH-recursion [9]. It is defined for any element $x \in \mathfrak{g}$ through the recursion

$$
\chi(t x):=t x+\overline{\mathrm{BCH}}\left(-\pi_{+}(\chi(t x)), t x\right) \in \mathcal{U}(\mathfrak{g})[[t]] .
$$

Remark 5.1. Note that $\chi(t x)$ is a formal power series whose coefficients are iterated commutators. For this reason it belongs to the vector subspace $\mathfrak{g}[[t]] \subset \mathcal{U}(\mathfrak{g})[[t]]$, whose elements are formal power series with coefficients in $\mathcal{U}(\mathfrak{g})$ of degree 1 .

The first few terms of the expansion $\chi(t x):=\sum_{n>0} t^{n} \chi_{n}(x)$ are given by

$$
\begin{aligned}
\chi(t x)= & t x-\frac{t^{2}}{2}\left[\pi_{+}(x), x\right]+\frac{t^{3}}{4}\left[\pi_{+}\left(\left[\pi_{+}(x), x\right]\right), x\right] \\
& +\frac{t^{3}}{12}\left(\left[\pi_{+}(x),\left[\pi_{+}(x), x\right]\right]-\left[\left[\pi_{+}(x), x\right], x\right]\right)+\cdots .
\end{aligned}
$$

Fix $a_{0} \in \mathfrak{g}$, and suppose that $\pi_{-}$is an $R$-matrix. In [9] it was shown, that (5.1) implies in $\mathcal{U}(\mathfrak{g})[[t]]$ the factorization

$$
\exp \left(t a_{0}\right)=\exp \left(\pi_{+}\left(\chi\left(t a_{0}\right)\right)\right) \exp \left(\pi_{-}\left(\chi\left(t a_{0}\right)\right)\right)
$$

Note that

$$
\exp \left(t a_{0}\right)=\exp \left(-\pi_{-}\left(\chi\left(-t a_{0}\right)\right)\right) \exp \left(-\pi_{+}\left(\chi\left(-t a_{0}\right)\right)\right)
$$

If $\mathfrak{g}=\mathfrak{g}_{+} \oplus \mathfrak{g}_{-}$, and if the corresponding projectors $\pi_{ \pm}: \mathfrak{g} \rightarrow \mathfrak{g}_{ \pm}$satisfy (2.1), then the above factorization is unique.

Recall that the binary product $x \triangleright y:=-\left[\pi_{+}(x), y\right]$ defines a post-Lie algebra structure on $\mathfrak{g}$, and

$$
\llbracket x, y \rrbracket=\left[\pi_{-}(x), y\right]+\left[x, \pi_{-}(y)\right]-[x, y]=x \triangleright y-y \triangleright x+[x, y]
$$

is the double Lie bracket (2.2).

Remark 5.2. In what follows we will work simultaneously with $\mathcal{U}(\mathfrak{g})[[t]]$ and $\mathcal{U}_{*}(\mathfrak{g})[[t]]$, which are the rings of formal power series with coefficients in $\mathcal{U}(\mathfrak{g})$ and $\mathcal{U}_{*}(\mathfrak{g})$, respectively. Note that these two rings are endowed with a natural Hopf algebra structure, inherited from $\mathcal{U}(\mathfrak{g})$ and $\mathcal{U}_{*}(\mathfrak{g})$. Furthermore, note that the last statement in Theorem 4.1 implies that $\mathcal{U}_{*}(\mathfrak{g})[[t]]$ is isomorphic, as a Hopf algebra, to $\mathcal{U}(\overline{\mathfrak{g}})[[t]]$.

From (5.2) and (4.2) we deduce the next proposition.

Proposition 5.3. For any $a \in \mathfrak{g}$, the following identity holds in $\mathcal{U}(\mathfrak{g})[[t]]$

$$
\exp (-t a)=\exp ^{*}(-\chi(t a))
$$

\footnotetext{
${ }^{1}$ Regarding item (iv) in Remark 4.2 we note that in this section we have decided to work with formal series in the parameter $t$. However, we could have taken a local point of view by assuming convergence of the BCH-series together with small enough $t$, which would imply that the exponential maps exp and exp* lie in sufficiently small neighborhoods of the units of the groups $G$ and $\bar{G}$, respectively, corresponding to the Lie algebras $\mathfrak{g}$ and $\overline{\mathfrak{g}}$.
} 
Proof. Statement (5.3) follows from (4.2) by considering $\chi(t a) \in \mathfrak{g}[[t]]$

$$
\exp ^{*}(-\chi(t a))=\exp \left(-\pi_{-}(\chi(t a))\right) \exp \left(-\pi_{+}(\chi(t a))\right) .
$$

Then (5.2) implies that $\exp (-t a)=\exp ^{*}(-\chi(t a))$.

From (5.3) we see that $\exp (t a)=\exp ^{*}(-\chi(-t a))$ in $\mathcal{U}(\mathfrak{g})[[t]]$. From Proposition 5.3 we get a differential equation for the BCH-recursion (5.1) in $\mathcal{U}(\mathfrak{g})[[t]]$ :

$$
\begin{aligned}
\operatorname{dexp}_{\chi(t a)}^{*}(\dot{\chi}(t a)) & =\exp ^{*}(\chi(t a)) *(\exp (-t a) a) \\
& =\exp ^{*}(\chi(t a))\left(\exp ^{*}(\chi(t a)) \triangleright(\exp (-t a) a)\right) \\
& =\exp ^{*}(\chi(t a))\left(\left(\exp ^{*}(\chi(t a)) \triangleright \exp (-t a)\right)\left(\exp ^{*}(\chi(t a)) \triangleright a\right)\right) \\
& =\exp ^{*}(\chi(t a))\left(\left(\exp ^{*}(\chi(t a)) \triangleright \exp ^{*}(-\chi(t a))\right)\left(\exp ^{*}(\chi(t a)) \triangleright a\right)\right) \\
& =\left(\exp ^{*}(\chi(t a))\left(\exp ^{*}(\chi(t a)) \triangleright \exp ^{*}(-\chi(t a))\right)\right)\left(\exp ^{*}(\chi(t a)) \triangleright a\right) \\
& =\left(\exp ^{*}(\chi(t a)) * \exp ^{*}(-\chi(t a))\right)\left(\exp ^{*}(\chi(t a)) \triangleright a\right)=\exp ^{*}(\chi(t a)) \triangleright a,
\end{aligned}
$$

and therefore

$$
\dot{\chi}(t a)=\operatorname{dexp}_{\chi(t a)}^{*-1}\left(\exp ^{*}(\chi(t a)) \triangleright a\right) .
$$

Theorem 5.4. The solution of the differential equation

$$
\dot{a}(t)=a(t) \triangleright a(t), \quad a(0)=a_{0}
$$

in $\mathcal{U}(\mathfrak{g})[[t]]$, is given by

$$
a(t):=\exp ^{*}\left(\chi\left(a_{0} t\right)\right) \triangleright a_{0} .
$$

Proof. From (5.4) we find that

$$
\exp ^{*}\left(\chi\left(a_{0} t\right)\right) \triangleright a_{0}=\operatorname{dexp}_{\chi\left(a_{0} t\right)}^{*}\left(\dot{\chi}\left(a_{0} t\right)\right)=a(t) .
$$

This yields

$$
\begin{aligned}
\dot{a}(t) & =\left(\operatorname{dexp}_{\chi\left(a_{0} t\right)}^{*}\left(\dot{\chi}\left(a_{0} t\right)\right) * \exp ^{*}\left(\chi\left(a_{0} t\right)\right)\right) \triangleright a_{0} \\
& =\operatorname{dexp}_{\chi\left(a_{0} t\right)}^{*}\left(\dot{\chi}\left(a_{0} t\right)\right) \triangleright\left(\exp ^{*}\left(\chi\left(a_{0} t\right)\right) \triangleright a_{0}\right)=a(t) \triangleright a(t) .
\end{aligned}
$$

Recall Proposition 4.4. It implies for $a_{0} \in \mathfrak{g}$ that

$$
a(t)=\exp ^{*}\left(\chi\left(a_{0} t\right)\right) \triangleright a_{0}=\exp \left(-\pi_{+}\left(\chi\left(a_{0} t\right)\right)\right) a_{0} \exp \left(\pi_{+}\left(\chi\left(a_{0} t\right)\right)\right) .
$$

We deduce that $a(t) \in \mathfrak{g}[[t]]$. Therefore, assuming that $t$ is sufficiently small and the BCHrecursion convergent, such that $\exp ^{*}\left(\chi\left(a_{0} t\right)\right)$ lies in a neighborhood of the unit of the group $G$ corresponding to the Lie algebra $\mathfrak{g}$, then $a(t)=\exp ^{*}\left(\chi\left(a_{0} t\right)\right) \triangleright a_{0}$ solves the Lie bracket equation in $\mathfrak{g}$

$$
\dot{a}(t)=\left[a(t), p_{+}(t)\right]
$$

with initial value $a(0)=a_{0}$, and $p_{+}(t)=\pi_{+}(a(t))$.

Note that

$$
\operatorname{dexp}_{x}^{*-1}(y)=\frac{\operatorname{ad}_{x}^{*}}{\exp \operatorname{ad}_{x}^{*}-1}(y)=\sum_{n \geq 0} \frac{B_{n}}{n !} \operatorname{ad}_{x}^{* n}(y),
$$


where $B_{n}$ are the Bernoulli numbers, and $\operatorname{ad}_{x}^{* n}(y):=\operatorname{ad}_{x}^{* n-1}(\llbracket x, y \rrbracket), \operatorname{ad}_{x}^{* 0}(y):=y$. Hence, with $\chi(t a):=\sum_{n>0} t^{n} \chi_{n}(a)$ it follows that

$$
\begin{aligned}
\operatorname{dexp}_{\chi\left(a_{0} t\right)}^{*-1} & :=\frac{\operatorname{ad}_{\left.\chi\left(a_{0} t\right)\right)}^{*}}{\exp \operatorname{ad}_{\chi\left(a_{0} t\right)}^{*}-1}=\sum_{s \geq 0} \frac{B_{s}}{s !} \operatorname{ad}_{\chi\left(a_{0} t\right)}^{* s} \\
& =1+\sum_{s>0} t^{s} \sum_{j=1}^{s} \frac{B_{j}}{j !} \sum_{\substack{k_{1}+\cdots+k_{j}=s \\
k_{i}>0}} \operatorname{ad}_{\chi_{k_{1}}}^{*} \cdots \operatorname{ad}_{\chi_{k_{j}}}^{*}
\end{aligned}
$$

The post-Lie exponential $\exp ^{*}\left(\chi\left(a_{0} t\right)\right) \triangleright a_{0}$ is defined through

$$
\begin{aligned}
\exp ^{\triangleright}\left(\chi\left(a_{0} t\right)\right) a_{0} & :=a_{0}+\sum_{i>0} \frac{1}{i !} \chi\left(a_{0} t\right) \triangleright\left(\chi\left(a_{0} t\right) \cdots \triangleright\left(\chi\left(a_{0} t\right) \triangleright a_{0}\right)\right) \\
& =a_{0}+\sum_{i>0} \frac{t^{i}}{i !} \sum_{u=1}^{i} \sum_{\substack{k_{1}+\cdots+k_{u}=i \\
k_{i}>0}} \chi_{k_{1}} \triangleright\left(\chi_{k_{2}} \triangleright \cdots\left(\chi_{k_{u}} \triangleright a_{0}\right)\right),
\end{aligned}
$$

which yields for $\Omega_{\triangleright}\left(a_{0} t\right):=\sum_{n>0} t^{n} \Omega_{n}\left(a_{0}\right)=\int_{0}^{t} \dot{\chi}\left(a_{0} s\right) d s$

$$
\Omega_{1}=a_{0}, \quad \Omega_{2}=\frac{1}{2} a_{0} \triangleright a_{0},
$$

and for $n>2$

$$
\begin{aligned}
n \Omega_{n}= & \sum_{j=1}^{n-1} \frac{1}{j !} \sum_{\substack{k_{1}+\cdots+k_{j}=n-1 \\
k_{i}>0}} \Omega_{k_{1}} \triangleright\left(\Omega_{k_{2}} \triangleright \cdots\left(\Omega_{k_{j}} \triangleright a_{0}\right)\right)+\sum_{j=1}^{n-1} \frac{B_{j}}{j !} \sum_{\substack{k_{1}+\cdots+k_{j}=n-1 \\
k_{i}>0}} \operatorname{ad}_{\Omega_{k_{1}}}^{*} \cdots \operatorname{ad}_{\Omega_{k_{j}}}^{*} a_{0} \\
& +\sum_{j=2}^{n-1}\left(\left(\sum_{s=1}^{j-1} \frac{B_{s}}{s !} \sum_{\substack{k_{1}+\cdots+k_{s}=j-1 \\
k_{i}>0}} \operatorname{ad}_{\Omega_{k_{1}}}^{*} \cdots \operatorname{ad}_{\Omega_{k_{s}}}^{*}\right)\right. \\
& \left.\times\left(\sum_{u=1}^{n-j} \frac{1}{u !} \sum_{\substack{k_{1}+\cdots+k_{u}=n-j \\
k_{i}>0}} \Omega_{k_{1}} \triangleright\left(\Omega_{k_{2}} \triangleright \cdots\left(\Omega_{k_{u}} \triangleright a_{0}\right)\right)\right)\right)
\end{aligned}
$$

Remark 5.5. This formula is equivalent to equation (14) in [6] (see also [5]), using $\left[\pi_{+}(x), \pi_{+}(y)\right]$ $=-\pi_{+}(\llbracket x, y \rrbracket)$, such that

$$
\pi_{+}\left(\operatorname{ad}_{\Omega_{k_{1}}}^{*} \cdots \operatorname{ad}_{\Omega_{k_{u}}}^{*} a_{0}\right)=(-1)^{u} \operatorname{ad}_{\Omega_{k_{1}}} \cdots \operatorname{ad}_{\Omega_{k_{u}}} \pi_{+}\left(a_{0}\right) .
$$

Theorem 5.6. Given $x \in \mathfrak{g}$, and $\xi \in \mathcal{U}(\mathfrak{g})$. Then in $\mathcal{U}(\mathfrak{g})[[t]]$

$$
\exp (t x) * \xi=\exp \left(-\pi_{-}(\chi(-t x))\right) \xi \exp \left(-\pi_{+}(\chi(-t x))\right) .
$$

Proof. First we calculate $\exp (t x) * \xi$ for $\xi \in \mathfrak{g}$. We find

$$
\exp (t x) * \xi=\exp (t x)(\exp (t x) \triangleright \xi)=\exp (t x)\left(\exp ^{*}(-\chi(-t x)) \triangleright \xi\right),
$$

where we used (5.3). Then

$$
\exp ^{*}(-\chi(-t x)) \triangleright \xi=\exp ^{\triangleright}(-\chi(-t x)) \xi=\exp \left(\pi_{+}(\chi(-t x))\right) \xi \exp \left(-\pi_{+}(\chi(-t x))\right) .
$$


Therefore

$$
\begin{aligned}
\exp (t x) * \xi & =\exp (t x)\left(\exp ^{*}(-\chi(-t x)) \triangleright \xi\right) \\
& =\exp \left(-\pi_{-}(\chi(-t x))\right) \exp \left(-\pi_{+}(\chi(-t x))\right) \exp \left(\pi_{+}(\chi(-t x))\right) \xi \exp \left(-\pi_{+}(\chi(-t x))\right) \\
& =\exp \left(-\pi_{-}(\chi(-t x))\right) \xi \exp \left(-\pi_{+}(\chi(-t x))\right) .
\end{aligned}
$$

Now we suppose that $\xi \in \mathcal{U}(\mathfrak{g})$. For notational simplicity we assume that $\xi=a^{n}, a \in \mathfrak{g}$.

$$
\begin{aligned}
\exp ^{*}(-\chi(-t x)) \triangleright a^{n} & =\exp ^{\triangleright}(\chi(-t x)) a^{n} \\
& =a^{n}-\chi(-t x) \triangleright a^{n}+\frac{1}{2 !} \chi(-t x) \triangleright\left(\chi(-t x) \triangleright a^{n}\right)+\cdots
\end{aligned}
$$

Recall that for $\chi(-t x) \in \mathfrak{g}[[t]]$

$$
\begin{aligned}
\chi(-t x) \triangleright a^{n} & =\sum_{i=1}^{n} a^{i-1}(\chi(-t x) \triangleright a) a^{n-i}=-\sum_{i=1}^{n} a^{i}\left[\pi_{+}(\chi(-t x)), a\right] a^{n-i} \\
& =-\left[\pi_{+}(\chi(-t x)), a^{n}\right] .
\end{aligned}
$$

The last Lie bracket belongs to $\mathcal{U}(\mathfrak{g})[[t]]$. This yields

$$
\chi(-t x) \triangleright a^{n}=\exp \left(\pi_{+}(\chi(-t x))\right) a^{n} \exp \left(-\pi_{+}(\chi(-t x))\right),
$$

which implies the result.

Theorem 5.7. For every $x, y \in \mathfrak{g}$,

$$
\exp (t x) * \exp (y)=\exp \left(-\pi_{-}(\chi(-t x))\right) \exp (y) \exp \left(-\pi_{+}(\chi(-t x))\right)
$$

and

$$
(\exp (t x))^{*-1}=\exp \left(\pi_{-}(\chi(-t x))\right) \exp \left(\pi_{+}(\chi(-t x))\right)
$$

Proof. Recall that we write $\exp v, \exp ^{*} v$ and $\exp v$ to denote the exponentials of $v$ in $\mathcal{U}(\mathfrak{g})$, $\mathcal{U}_{*}(\mathfrak{g})$ and $\mathcal{U}(\overline{\mathfrak{g}})$, respectively.

Identity (5.5) follows from (5.3), and applying the map $F$ to

$$
\exp (-\chi(-t x)) \cdot \exp (-\chi(-t x)) \in \mathcal{U}(\overline{\mathfrak{g}})[[t]]
$$

The morphism property of $F$ yields

$$
\begin{aligned}
& F(\exp (-\chi(-t x)) \cdot \exp (-\chi(-t x))) \\
& \quad=\exp ^{*}(-\chi(-t x)) * \exp ^{*}(-\chi(-t x))=\exp (t x) * \exp (y),
\end{aligned}
$$

and

$$
\begin{aligned}
& F(\exp (-\chi(-t x)) \cdot \exp (-\chi(-t x))) \\
& \quad=\exp \left(-\pi_{-}(\chi(-t x))\right) \exp \left(-\pi_{-}(\chi(-t y))\right) \exp \left(-\pi_{+}(\chi(-t y))\right) \exp \left(-\pi_{+}(\chi(-t x))\right) \\
& \quad=\exp \left(-\pi_{-}(\chi(-t x))\right) \exp (y) \exp \left(-\pi_{+}(\chi(-t x))\right) .
\end{aligned}
$$




\section{Conclusions}

We have addressed the problem of finding explicit solutions to isospectral flow equations. These form a class of differential equations usually encountered in the theory of (Hamiltonian) dynamical systems, and commonly studied using methods borrowed from Lie theory. In general, their solutions are obtained starting from the existence of a solution of the modified classical YangBaxter equation. In other words, from the existence of a classical $R$-matrix on the underlying Lie algebra.

We have outlined how to approach the problem using a framework based on a particular class of non-associative algebras known as post-Lie algebras. More precisely, starting from a classical $R$-matrix solving the modified classical Yang-Baxter equation on a (finite-dimensional) Lie algebra $\mathfrak{g}$, we showed how the corresponding post-Lie algebra structure on the Lie enveloping algebra $\mathcal{U}(\mathfrak{g})$ allows us to describe explicit solutions of exponential type to any isospectral flow equation defined on the original Lie algebra $\mathfrak{g}$.

\section{Acknowledgements}

We acknowledge support from ICMAT and the Severo Ochoa Excelencia Program, as well as the NILS-Abel project 010-ABEL-CM-2014A, and the SPIRIT project RCN:231632 of the Norwegian Research Council. The first author is supported by a Ramón y Cajal research grant from the Spanish government, and acknowledges support from the Spanish government under project MTM2013-46553-C3-2-P.

\section{References}

[1] Babelon O., Bernard D., Talon M., Introduction to classical integrable systems, Cambridge Monographs on Mathematical Physics, Cambridge University Press, Cambridge, 2003.

[2] Bai C., Guo L., Ni X., Nonabelian generalized Lax pairs, the classical Yang-Baxter equation and PostLie algebras, Comm. Math. Phys. 297 (2010), 553-596, arXiv:0910.3262.

[3] Burde D., Left-symmetric algebras, or pre-Lie algebras in geometry and physics, Cent. Eur. J. Math. 4 (2006), 323-357, math-ph/0509016.

[4] Cartier P., Vinberg algebras, Lie groups and combinatorics, in Quanta of Maths, Clay Math. Proc., Vol. 11, Amer. Math. Soc., Providence, RI, 2010, 107-126.

[5] Casas F., Numerical integration methods for the double-bracket flow, J. Comput. Appl. Math. 166 (2004), 477-495.

[6] Casas F., Iserles A., Explicit Magnus expansions for nonlinear equations, J. Phys. A: Math. Gen. 39 (2006), $5445-5461$.

[7] Chapoton F., Livernet M., Pre-Lie algebras and the rooted trees operad, Int. Math. Res. Not. 2001 (2001), no. 8, 395-408, math.QA/0002069.

[8] Chu M.T., Norris L.K., Isospectral flows and abstract matrix factorizations, SIAM J. Numer. Anal. 25 (1988), 1383-1391.

[9] Ebrahimi-Fard K., Guo L., Manchon D., Birkhoff type decompositions and the Baker-Campbell-Hausdorff recursion, Comm. Math. Phys. 267 (2006), 821-845, math-ph/0602004.

[10] Ebrahimi-Fard K., Lundervold A., Munthe-Kaas H.Z., On the Lie enveloping algebra of a post-Lie algebra, J. Lie Theory 25 (2015), 1139-1165, arXiv:1410.6350.

[11] Faibusovich L.E., QR-type factorizations, the Yang-Baxter equation, and an eigenvalue problem of control theory, Linear Algebra Appl. 122-124 (1989), 943-971.

[12] Lax P.D., Outline of a theory of the KdV equation, in Recent Mathematical Methods in Nonlinear Wave Propagation (Montecatini Terme, 1994), Lecture Notes in Math., Vol. 1640, Springer, Berlin, 1996, 70-102.

[13] Manchon D., A short survey on pre-Lie algebras, in Noncommutative Geometry and Physics: Renormalisation, Motives, Index Theory, ESI Lect. Math. Phys., Eur. Math. Soc., Zürich, 2011, 89-102. 
[14] Munthe-Kaas H.Z., Lundervold A., On post-Lie algebras, Lie-Butcher series and moving frames, Found. Comput. Math. 13 (2013), 583-613, arXiv:1203.4738.

[15] Oudom J.-M., Guin D., On the Lie enveloping algebra of a pre-Lie algebra, J. K-Theory 2 (2008), 147-167, math.QA/0404457.

[16] Reshetikhin N.Yu., Semenov-Tian-Shansky M.A., Quantum $R$-matrices and factorization problems, J. Geom. Phys. 5 (1988), 533-550.

[17] Semenov-Tjan-Shanskii M.A., What is a classical r-matrix?, Funct. Anal. Appl. 17 (1983), 259-272.

[18] Semenov-Tjan-Shanskii M.A., Classical r-matrix and quantization, J. Sov. Math. 31 (1985), 3411-3416.

[19] Suris Yu.B., The problem of integrable discretization: Hamiltonian approach, Progress in Mathematics, Vol. 219, Birkhäuser Verlag, Basel, 2003.

[20] Sweedler M.E., Hopf algebras, Mathematics Lecture Note Series, W.A. Benjamin, Inc., New York, 1969.

[21] Vallette B., Homology of generalized partition posets, J. Pure Appl. Algebra 208 (2007), 699-725, math.AT/0405312.

[22] Watkins D.S., Isospectral flows, SIAM Rev. 26 (1984), 379-391. 